\title{
Electroacupuncture upregulates ERK signaling pathways and promotes adult hippocampal neural progenitors proliferation in a rat model of depression
}

\author{
Liu Yang, Na Yue, Xiaocang Zhu, Qiuqin Han, Qiong Liu, Jin Yu* and Gencheng Wu*
}

\begin{abstract}
Background: In this study, we investigate the proliferation of adult neural stem cells (NSCs) in a chronic unpredictable stress (CUS) rat model of depression, the effects of electroacupunture (EA) on depressive-like symptoms and the corresponding signaling pathways.

Methods: SD rats were subjected to 4 weeks of CUS to induce depressive-like behaviors. EA was performed at the Du-20 (Bai-Hui) and GB-34 (Yang-Ling-Quan) acupoints. Rats were injected with BrdU and the brains were cut into sections. Double-labeling with BrdU/Sox2 and p-ERK/Nestin was performed to demonstrate the in vivo proliferation of adult NSCs in hippocampus and ERK activation in NSCs. Hippocampal microdialysates of different groups were collected to observe the in vitro effects on NSCs.

Results: After 8 treatments, EA generated a clear antidepressant effect on the stressed rats and promoted the NSC proliferation. ERK activation might be involved in the antidepressant-like effects of EA treatment. Hippocampal microdialysates from EA-treated stressed rats influenced NSCS to form larger neural spheres and exhibit higher p-ERK level in vitro, compared to the untreated stressed rats. Meanwhile, the antidepressant-like effects of EA involved contribution from both acupoint specificity and electrical stimulus.
\end{abstract}

Conclusions: EA might interfere with the hippocampal microenvironment and enhance the activation of ERK signaling pathways. This could mediate, at least in part, the beneficial effects of EA on NSC proliferation and depressive-like behaviors.

Keywords: Electroacupuncture, Depression, Neural stem cell, p-ERK, Hippocampal dentate gyrus, Microdialysis

\section{Background}

Electroacupuncture (EA) is one of the most frequently used therapeutic modalities for mental disorders, particularly in the East [1,2]. A growing number of studies have demonstrated the antidepressant-like effects of EA $[3,4]$. However, the neural mechanisms underlying the antidepressant-like effects of EA on depression remain complicated. One potential mechanism which has been repeatedly mentioned is that EA is beneficial to adult

\footnotetext{
* Correspondence: yujin@shmu.edu.cn; gcwu@shmu.edu.cn Department of Integrative Medicine and Neurobiology, Institute of Acupuncture Research (WHO Collaborating Center for Traditional Medicine), State Key Lab of Medical Neurobiology, Institutes of Brain Science, Shanghai Medical College, Fudan University, Shanghai 200032, P.R. China
}

neural stem cell (NSC) survival and proliferation, which underlies antidepressant-like effects of EA or classical antidepressants $[5,6]$. Nevertheless, the signaling pathways involved in the effects of EA on the proliferation of NSCs remain largely unexplored.

Various growth/neurotrophic factors, such as nerve growth factor (NGF), brain-derived neurotrophic factor (BDNF) and glial cell line-derived neurotrophic factor (GDNF), have been shown to mediate the survival of neurons and the proliferation of NSCs $[7,8]$. While several studies have indicated that EA raises the levels of these growth/neurotrophic factors $[9,10]$, the extracellular signal-regulated kinase (ERK) has been demonstrated to mediate the effects of these factors on adult 
neurogenesis $[1,10]$. Therefore, it is possible that EA improves the depressive-like behaviors and activates NSCs through signaling pathways including ERK.

At present, we explored the proliferative effect of EA on adult NSCs, and then investigated the level of phosphorylated extracellular signal-regulated kinase (p-ERK) of hippocampal NSCs in a chronic unpredictable stress (CUS) rat model of depression. In addition, to determine whether EA results in microenvironment change that is conducive to NSC proliferation in the dentate gyrus (DG), we observed the effects of microdialysates from the DG of CUS rats with/without EA treatment on the proliferation of NSCs in vitro. The results exhibited that the growth of neurospheres and p-ERK levels in NSCs were up-regulated by microdialysates from the EAtreated stressed rats. Taken together, the antidepressantlike action of EA on the CUS model might be associated with the extracellular microenvironment of hippocampal NSCs.

\section{Methods \\ Animals}

The experimental protocol and procedures used in this study were both in strict accordance with the National Institutes of Health Guide for the Care and Use of Laboratory Animals and approved by the Experimental Animal Ethics Committee of Shanghai Medical College (Fudan University). Sprague-Dawley rats (male, $220 \mathrm{~g}$ ) were housed in individual cages, at $20-22^{\circ} \mathrm{C}$ with a $12 / 12 \mathrm{~h} \mathrm{light} /$ dark cycle and with free access to food and water.

\section{CUS-induced depression model}

SD rats were subjected to 4 weeks of CUS to induce depressive-like behaviors $[4,11]$. Seven types of stressors were used (40-h water deprivation, 40-h food deprivation, revered light-dark-cycle, 5-min hot environment, 5-min swimming in cold water, 30-min cage shake and wet cage) in a semi-random order. Rats were exposed to one or two stressors every two days (the next stressor appeared on the next day or two days later). Every type of stressors would appear at least once in the first 2 weeks. Nineteen times of stressors were used in all.

\section{EA delivery}

The stressed rats in EA group started to receive EA on day 15 and were treated for 2 weeks (the latter part of the CUS period, 8 times, once every other day). EA stimulation was performed at 'Bai-Hui' $(\mathrm{Du}-20)(+)$ and 'Yang-Ling-Quan' (GB-34, the right side) (-) acupoints. These two acupoints have been applied and showed some antidepressant effects in our preliminary and other studies [12-15]. The rat was moderately bound by a piece of self-made clothing and hung approximately
$0.15 \mathrm{~m}$ high, under halothane anesthesia. A pair of stainless steel needles was connected to the output terminals of the EA apparatus (LH202H, HANS Electronic Apparatus, $2 \backslash 100 \mathrm{~Hz}, 0.3 \mathrm{~mA}$, lasted for $30 \mathrm{~min}$ ). Taken together, the rats were divided into three groups $(\mathrm{n}=7 \sim 8$ for each group): Normal, Model (i.e. CUS) and EA (i.e. CUS + EA) groups.

\section{Behavioral tests}

We used the forced swimming test (FST) and elevated plus-maze (EPM) to determine the depression degree, as extensively observed in many studies. The FST was performed on the first day of every week. Briefly, the rats were individually placed in a glass cylinder $(30 \mathrm{~cm}$ of diameter) containing $25 \mathrm{~cm}$ of fresh water. A fifteen min adaptation of the water was performed one day prior to the first test. The total durations of immobility time and climbing time within the 5 min test period were recorded. An increased immobility time or decreased climbing time was indicative of depressive-like behavior. In the EPM test, the rats were allowed to move freely for $5 \mathrm{~min}$ in the apparatus. Percent entries into and time in open arms were related to fear of novelty. Measures were: (1) percentage open-arms entries ([open-arms entries/(open-arms + enclosed-arms entries) $\times 100 \%$ ) and (2) percentage open-arms time ([open-arms time/(totalarms time) $] \times 100 \%$ ). We tested these two indices before CUS, in the second, third and fourth week of CUS by different sets of animals.

\section{BrdU injection}

To examine the proliferation of hippocampal progenitor cells, rats were injected with 5-bromo-2-deoxyuridine (BrdU; $100 \mathrm{mg} / \mathrm{kg}$, i.p.) on the final day of the 4-week period and were sacrificed $24 \mathrm{~h}$ after injection. The short survival time following BrdU injection allowed us to determine the effect of different manipulations on the proliferation rate of progenitor cells.

\section{Perfusion and tissue storage}

Rats were anesthetized with chloral hydrate $(350 \mathrm{mg} / \mathrm{kg})$ and transcardially perfused with $200 \mathrm{ml}$ normal saline followed by $300 \mathrm{ml} 4 \%$ paraformaldehyde (PFA) in $0.1 \mathrm{M}$ phosphate-buffer ( $\mathrm{PB}, \mathrm{pH}$ 7.4). The brains were removed from the skulls, and postfixed with $4 \%$ PFA in $0.1 \%$ PB for $24 \mathrm{~h}$ at $4^{\circ} \mathrm{C}$. Following post-fixation, the brains were immersed in $30 \%$ sucrose in $\mathrm{PB}$ for $48 \mathrm{~h}$ at $4^{\circ} \mathrm{C}$, and then, serial coronal sections of the brains were cut through the entire hippocampus into $30 \mu \mathrm{m}$ sections on a freezing microtome (Leica CM1900, Germany) and stored in cryoprotectant (25\% ethylene glycol, $25 \%$ glycerol, $0.05 \mathrm{M} \mathrm{PB}$, $\mathrm{pH} 7.4)$ at $-20^{\circ} \mathrm{C}$ until use. 


\section{Immunohistochemical analysis}

To investigate the proliferation of NSCs in DG, we examined BrdU-labeled Sox2-positive cells in the coronal brain sections. Sox 2 is a marker for stem/progenitor cells, and BrdU is used to characterize the cells with mitotic activity. Free-floating brain sections were processed for BrdU immunohistochemistry. The brain sections were incubated for $30 \mathrm{~min}$ in $2 \mathrm{M} \mathrm{HCl}$ at $37^{\circ} \mathrm{C}$, followed by several PBA rinses. After blocked/permeabilized for $1 \mathrm{~h}$ in PBS containing 4\% normal horse serum, 1\% BSA (Sigma-Aldrich, USA) and $0.3 \%$ Triton $\mathrm{X}-100$ at $37^{\circ} \mathrm{C}$, the sections were incubated with sheep anti-BrdU (1:100, Abcam, UK) and rabbit anti-Sox2 (1:200, Sigma-Aldrich, USA) overnight at $4^{\circ} \mathrm{C}$. Next, the sections were transferred into the secondary antibody solution (donkey anti sheep, R-Phycoerythrin conjugated, 1:200, Jackson ImmunoResearch, USA; donkey anti rabbit, Alexa 488 conjugated, 1:200, Invitrogen, USA; Hoechst, 1: 1000, Beyotime, China) and incubated for $1 \mathrm{~h}$ in the dark. The sections were then washed 5 times with PBS, covered with a coverslip, and sealed with $75 \%$ glycerol.

For Nestin and p-ERK double-staining, the protocol was similar except that $\mathrm{HCl}$ is not used and the antibodies was changed to rabbit anti p-ERK1/2 (1:200, Invitrogen, Life Technologies Inc., USA; corresponding secondary antibody: goat-anti-rabbit-Dylight 488, 1:200, Invitrogen, Life Technologies Inc., USA) and mouse-antiNestin (1:200, millipore, USA; corresponding secondary antibody: goat-anti-mouse-Alexa 680, 1:200, Invitrogen, Life Technologies Inc., USA).

The hippocampal DG region was examined under a fluorescence microscope or laser confocal microscope. For quantification, we counted the average number of positive cells per section in the DG zone from 3 brain sections of a rat and the data are presented as the average number per rat ( $n=5-6$ per group). Every two adjacent sections selected were $120 \sim 210 \mu \mathrm{m}$ apart (distance of $4 \sim 7$ sections), and the mean location of the 3 sections was approximately fontanel $-3.9 \mathrm{~mm}$.

\section{Hippocampal microdialysates}

After five EA treatments, the hippocampal microdialysates were collected from the rats in Model and EA groups separately. The microdialysis-intracerebral-guidecannula (Bioanalytical Systems, Inc., West Lafayette, USA) was implanted into the hippocampal DG zone with the following coordinates: $3.9 \mathrm{~mm}$ posterior and $1.5 \mathrm{~mm}$ lateral to bregma; $3.9 \mathrm{~mm}$ ventral to the cortical surface. The guide cannula was fixed to the skull surface with Duralay dental cement. A microdialysis probe with a $2 \mathrm{~mm}$ membrane (Bioanalytical Systems, Inc., West Lafayette, USA) was then placed through the guide cannula into the DG region. Physiological saline was applied as the perfusate. The optimal perfusion flow-rate was set at $2.5 \mu \mathrm{l} / \mathrm{min}$ and the sampling time for each rat was $2.5 \mathrm{~h}$ (approximately 300-350 $\mathrm{\mu l}$ of microdialysate was obtained, in addition to the loss of the leading portion from the collecting canal).

\section{NSC culture under the influence of microdialysates}

The hippocampus isolated from a postnatal day 0 (P0) SD rat was washed twice and mechanically dissociated into single cells by gentle trituration through a Pasteur pipette in aseptic PBS. The cell suspension was then centrifuged, resuspended, plated in low attachment dishes in NSC-conditioned medium and finally maintained in an incubator $\left(5 \% \mathrm{CO}_{2} /\right.$ air, at $\left.37^{\circ} \mathrm{C}\right)$. Components of the media were as follows: DMEM-F-12 medium containing epidermal growth factor (EGF, $20 \mathrm{ng} / \mathrm{ml}$, Invitrogen, Life Technologies Inc., USA), basic fibroblast growth factor (b-FGF, $20 \mathrm{ng} / \mathrm{ml}$, Invitrogen, Life Technologies Inc., USA), and B27 supplements (1:50; Invitrogen, Invitrogen, Life Technologies Inc., USA). The media was changed every $48 \mathrm{~h}$ (by centrifugation and resuspension of the spheres). After 9 days of cultivation, we observed the appearance of neurospheres under an optical microscope and detected the expression of Nestin with immunofluorescence (detailed procedures not shown, similarly performed according to universal protocols).

The purified NSCs were then cultured in different media (PBS group: 70\% NSC medium + 30\% PBS; Model group: $70 \%$ NSC medium $+30 \%$ microdialysates from CUS model rats; EA group: $70 \%$ NSC medium $+30 \%$ microdialysates from the EA-treated CUS rats). Before adding into the culture media, the microdialysates of all of the groups were aseptically filtered separately. Then, NSCs were mixed with different media and dissociated by pipette aspiration and inoculated into a 96-well plate $\left(5 \sim 5.5 \times 10^{3}\right.$ cells $/ 120 \mu \mathrm{l}$ medium/well $)$. After 3 days, another $100 \mu \mathrm{l}$ of corresponding medium was added to each well and then, after another 2 days, the other $80 \mu \mathrm{l}$ of corresponding medium was further supplied. The cell viability and growth of the NSC spheres were investigated $48 \mathrm{~h}$ after the second supplementation of the culture media.

For cell viability, $100 \mu \mathrm{l}$ of the cell suspension was transferred into a 96-well plate. CCK-8 (10 $\mu \mathrm{l}$, Dojindo Molecular Technologies, Inc. Japan) solution was added to each well. The plate was incubated at $37^{\circ} \mathrm{C}$ for $2 \mathrm{~h}$. The absorbance value at the $450 \mathrm{~nm}$ wavelength was measured with an ELx800 Absorbance Microplate Reader (BioTek Instruments, Inc. USA). The experiment was replicated 3 times with quadruplicate samples included in each experiment. The cell viability of the Model and EA groups were converted into a percentage of the PBS group [Cell-Viability ${ }_{\text {Model }} \%=\left(\right.$ Absorbance $_{\text {Model }^{-}}$ Absorbance $\left._{\text {Blank }}\right) /\left(\right.$ Absorbance $_{\text {PBS }}-$ Absorbance $\left._{\text {Blank }}\right) \times 100 \%$; 
Cell-Viability ${ }_{\mathrm{EA}} \%=\left(\right.$ Absorbance $_{\mathrm{EA}^{-}}-$Absorbance $\left._{\text {Blank }}\right) /$ (Absorbance ${ }_{\text {PBS }}-$ Absorbance $_{\text {Blank }}$ ) $\left.\times 100 \%\right]$.

Another $100 \mu \mathrm{l}$ NSC suspension was removed to count the number of spheres greater than $100 \mu \mathrm{m}$ in diameter. This number reflected the NSC-sphere-promoting capacity of the different mediums. In additon, an immunofluorescence assay was employed to detect p-ERK in cultured NSCs in different meida. Briefly, the other $100 \mu \mathrm{l}$ NSC suspension was transferred to another polylysine-treated 96-well plate and fixed in 4\% PFA. The following antibodies: rabbit anti p-ERK1/2 (primary, 1:200, Invitrogen, Life Technologies Inc., USA) goatanti-rabbit-Dylight 488 (secondary, 1:200, Invitrogen, Life Technologies Inc., USA) were used in immunocytochemical staining. The mean number of immunopositive cells in every well was counted in four non-overlapping visual fields at a magnification of $10 \times$ objective lens. The average was calculated for groups of three wells. And the final results are representative of at least three independent experiments.

\section{Statistical analysis}

Results are expressed as means \pm S.E.M. One-way analysis of variance was used in most cases to check statistical tendencies. Differences between two groups were analyzed by Student's $t$ test. $P<0.05$ was considered statistically significant. For NSCs cultures, the numerical data were collected from at least three independent experiments.

\section{Results and discussion Results}

\section{Antidepressant-like behavioral effects of EA on the} CUS-induced depression model

Before the CUS procedure, there was no significant difference among the groups according to the FST data. After 2 weeks of CUS, the stressed groups showed significant depressive-like behaviors (increased immobility, $\mathrm{F}_{2,45}=3.874, P<0.05$, respectively; and decreased climbing, $F_{2}, 45=4.020, P<0.05$, respectively, Figure $1 \mathrm{~A} \& \mathrm{~B}$ ). One to two weeks of EA treatment significantly decreased the immobility and promoted climbing of the stressed rats (immobility time: in the $3^{\text {rd }}$ week $\mathrm{F}_{2,22}=$ $22.90, P<0.01$ vs. Model, respectively; in the $4^{\text {th }}$ week $\mathrm{F}_{2}$, $22=23.10, P<0.01$ vs. Model, respectively; climing time: in the $3^{\text {rd }}$ week $\mathrm{F}_{2}, 22=13.24, P<0.01$ vs. Model, respectively; in the $4^{\text {th }}$ week $\mathrm{F}_{2,22}=10.24, P<0.01$ vs. Model, respectively, Figure 1A \& B). The EPM test revealed a significant decrease in open arm entries and time spent in open arms after 2 weeks of CUS which indicated the anxiety-like behavior of stressed rats $\left(\mathrm{F}_{2,45}=\right.$ $2.785, P<0.05$ vs. Normal, respectively; time: $F_{2,} 45=$ 6.896, $P<0.01$ vs. Normal; respectively, Figure $1 C \& D)$. EA treatment also alleviated anxiety-like behavior of
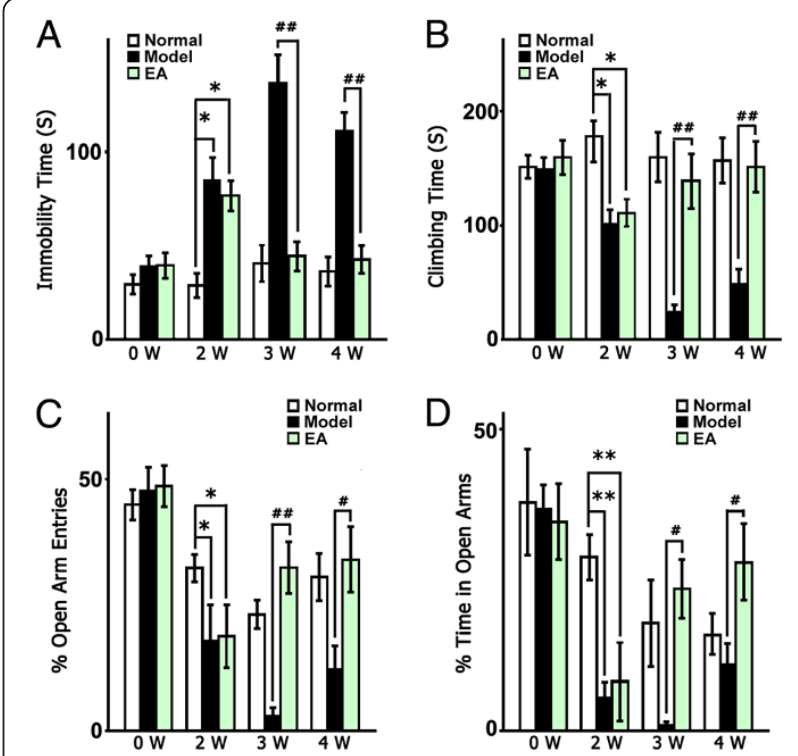

Figure 1 EA reversed the depressive-like behavior of the stressed rats. The normal group underwent no stress; the Model group received the chronic unpredictable stress (CUS) for 4 weeks; the EA group received CUS for 4 weeks, as well as EA treatment for the latter part of the CUS (2 weeks). (A) The immobility times in the forced swimming test (FST) decreased after 3 and 4 weeks of CUS (1 or 2 weeks of treatment) in the EA group compared with the Model group. (B) The climbing time in the FST was reversed to normal levels by EA treatment. (C) \& (D) The percentage of open-arms entries and open-arms time in the elevated plus maze (EPM). The values are presented as the mean $\pm S E, n=8$ or 18 in each group. Significance of "Model vs Normal" is marked by * $(P<0.05)$ or ** $(P<0.01)$, and that of "EA vs. Model" is marked by \# $(P<0.05)$ or \#\# $(P<0.01)$.

stressed rats, as indicated by increased open arm entries and time spent in open arms (in the $3^{\text {rd }}$ week: $F_{2}, 21=$ 8.21, $P<0.01$ vs. Model; $F_{2,2}=5.511, P<0.05$ vs. Model, respectively; in the $4^{\text {th }}$ week: $F_{2,47}=4.569, P<0.05$ vs. Model; $F_{2,47}=2.555, P<0.05$ vs. Model; respectively, Figure $1 C \& D)$.

\section{EA treatment promotes hippocampal stem cell proliferation in vivo}

Immunohistochemiscal assay results showed that the number of BrdU+/Sox $2+$ cells in hippocampus was significantly decreased in the stressed rats compared to that in the normal rats after 4 weeks of CUS procedure $\left(F_{2,15}=11.45, P<0.01\right.$ vs. Normal, Figure $\left.2 \mathrm{~A} \& \mathrm{~B}\right)$. Contrarily, the number of BrdU+/Sox2+ cells in hippocampus was significantly increased in EA-treated stressed rats in comparisons to the stressed rats $(P<0.05$ vs. Model, Figure 2A \& B).

\section{EA activates ERK1/2 phosphorylation in hippocampal stem cells in vivo}

Immunohistochemical assay results also indicated a similar relationship between EA and the activation of ERK1/2 


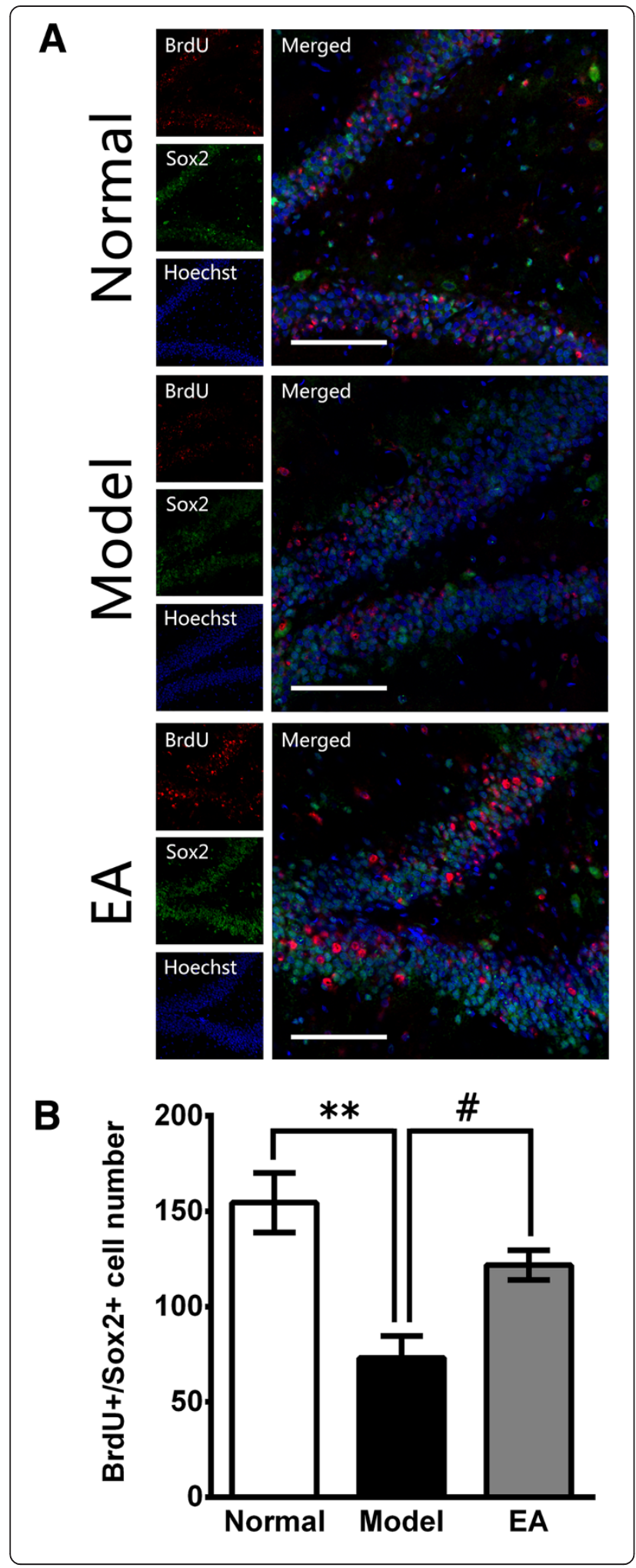

Figure 2 The effects of EA on stem cell proliferation in the hippocampal dentate gyrus (DG) after 4 weeks. (A) Representative triple immunofluorescence staining for BrdU + (red), Sox $2+$ (green) and Hoechst (blue) in the central region of the DG. Scale bar $=100 \mu \mathrm{m}$. (B) Quantitative representation of Sox2/BrdU double-positive cells in the DG zone (not only those regions in A) per section. EA treatment significantly improved the stem cell proliferation compared with the CUS Model. The values are the mean $\pm \mathrm{SE}, \mathrm{n}=5$ in each group. ${ }^{* *} P<0.01$ (Model vs. Normal); \# $P<0.05$ (EA vs. Model).

in stem cells. The number of p-ERK/Nestin doublepositive cells in the DG was greater in the EA-treated stressed rats compared to the stressed rats $\left(F_{2,15}=19.10\right.$, $P<0.01$ vs. Model, Figure 3A \& B). These results show that the MAPK/ERK signaling cascade in NSCs was activated in the EA-treated stressed rats.

\section{EA microdialysates up-regulated the neurosphere growth and $p$-ERK level of NSCs in vitro}

To determine the microenvironmental effect of EA, the proliferation and p-ERK level of NSCs were checked in different media. And after 7 days of culture in different media, the\% cell viability of the EA group was similar to the Model $(P>0.05$ vs. Model, Figure 4A). However, in the EA medium, the number of neurospheres whose diameters were greater than $100 \mu \mathrm{m}$ was more than that in the Model medium $(P>0.05$ vs. Model, Figure 4B). The number of large neurospheres indicates the tendency to form neurospheres and the maintenance of NSC properties (stem-like properties or stemness). These results suggest there may be dialyzable factors that promote neurosphere formation in the extracellular fluid of the hippocampal DG in the EA stimulated animals. Finally, we analyzed the p-ERK level of NSCs in vitro and found significantly more p-ERK-positive stem cells (visual fields under a $10 \times$ objective lens) in the EA medium (EA vs. Model $P<0.05$, Figure 4C). Therefore, the factors in the hippocampal microenvironment of the EA group may up-regulate the ERK signaling pathway in NSCs. These results are consistent with the in vivo findings and may potentially explain those findings about the antidepressant effects of EA.

\section{Antidepressant-like effects of EA involved contribution from both acupoint specificity and electrical stimulus}

To clarify the complicated mechanisms of the Antidepressant-like effects generated by EA, we hypothesized the action of EA involved two parts with separate mechanisms: acupoint specificity and electrical stimulus. Hence, we set a new group named Acupuncture, which was administered similar to the EA group except electrification. Changes produced in this group correspond to the therapeutic effects of physical stimulus at acupoints. After five treatments (began at day 15, after the $3^{\text {rd }}$ week 


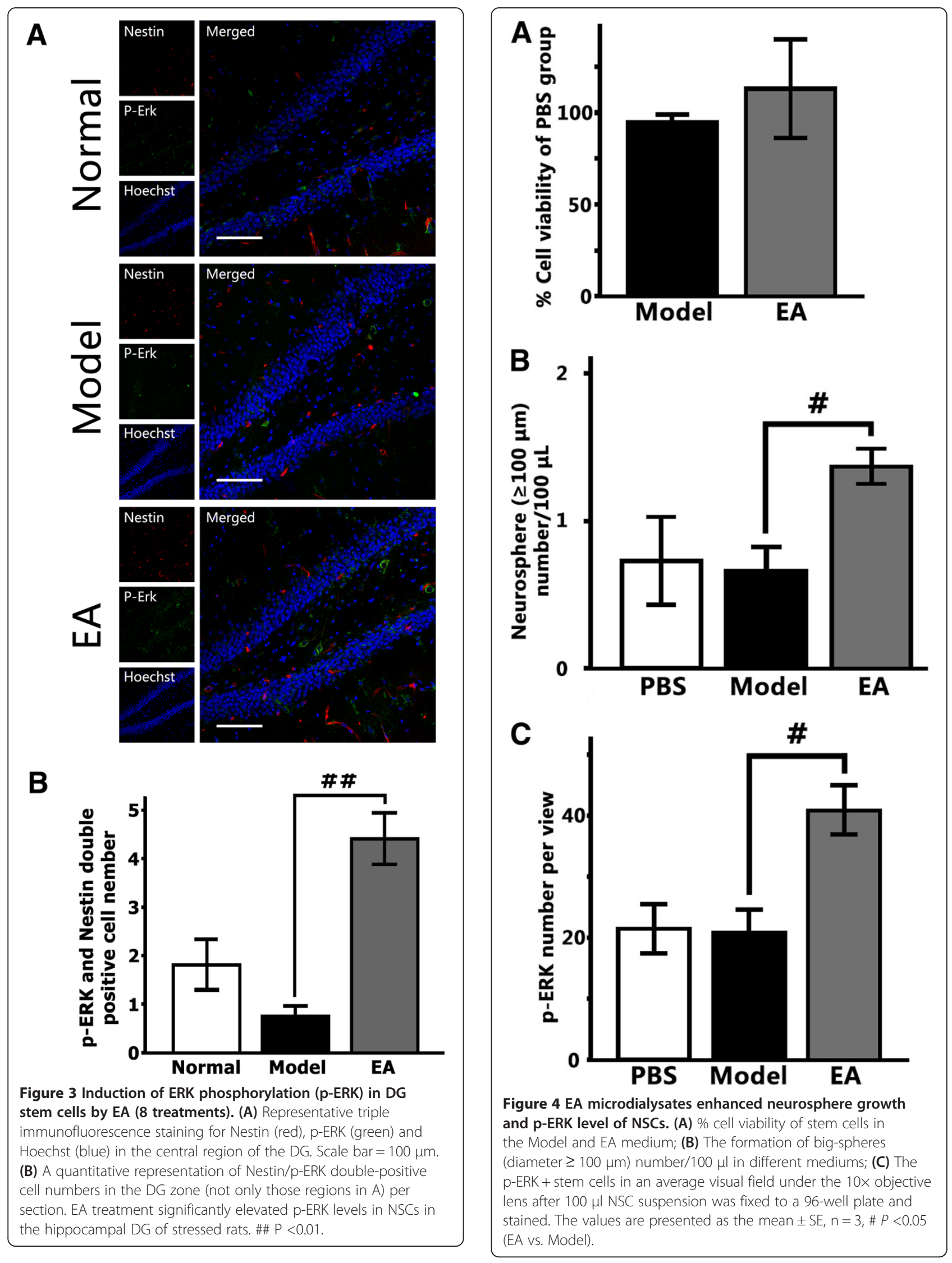


of CUS), Acupuncture significantly exhibited some antidepressant-like effects on the immobility time in the FST and open-arms entries in the EPM, without significant changes in the climbing time and open-arms time. Meanwhile, EA showed much more significant antidepressant-like effects which were evaluated by the significant changes in the immobility time and the climbing time in the FST and open-arms time and entries in the EPM (Figure 5, A: $F_{2,22}=15.31, P<0.001$ Acupuncture vs. Model, $P<0.0001$ EA vs. Model; $B$ : $F_{22} 22=12.26$, $P<0.001$ EA vs. Model, $P<0.01$ EA vs. Acupuncture; C: $F_{2,} 22=9.049, P<0.05$ Acupuncture vs. Model, $P<0.001$ EA vs. Model; $\mathrm{D}: \mathrm{F}_{2,22}=6.210, P<0.01 \mathrm{EA}$ vs. Model). These data support that the antidepressantlike effect could be independent of the electrical stimulus and strongly indicate that two factors, acupoint specificity and electrical stimulus, are both making contribution to the behavioral effects of EA.

\section{Discussion}

The major aim of the present study was to investigate the underlying microenvironmental mechanisms involved in the antidepressant-like efficacy of EA in the CUS model of depression. EA exhibited antidepressant-like effects and promoted the proliferation of adult hippocampal NSCs. The underlying mechanism may be related, at least partially, to the up-regulation of extracellular factors in the microenvironment of DG, and then induction of ERK phosphorylation in NSCs.

There have been clinical trials suggesting that EA treatment is an effective therapeutic strategy for depression
$[4,16]$. Consistent with these results, our experiments revealed the antidepressant-like effect of EA in the FST and EPM tests. At the meantime, our findings provided the evidence that an enough therapeutic level of EA included the action from stimulus at specific acupoints and the contribution from the electric current. However, acupoint specificity can also play a lower but significant role, independent of the electrical stimulus. It has been supported that acupuncture of such acupoints as Du-20, GB-34 and othe special acupoints has specific antidepressant-like effect by the traditional Chinese medical theories and many published references $[4,12,14,15,17,18]$. To date, the underlying mechanism of acupoint specificity is not fully revealed. One possible interpretation could be as follows. Normally, there are more nerves distributed in acupoints than adjacent skin areas. Therefore more nerve receptors are activated during acupuncture treatment at acupoints compared to adjacent skin areas. Electroacupuncture also produce electrical stimulus to these receptors. The electrical stimulus also contributed to the antidepressant-like effects which were more stabilized and significant. However, exact mechanism merits further study.

Recently, studies on depression have focused on neuroprotection and NSC-proliferation in DG, and an increased number of studies support the hypothesis that acupuncture can be an effective treatment for depression and a rescuer of impaired neurogenesis [19-21]. Our results provide further evidence in supporting this hypothesis.

Although, whether ablation of NSC proliferation or neurogenesis elicits a depression-like phenotype is still
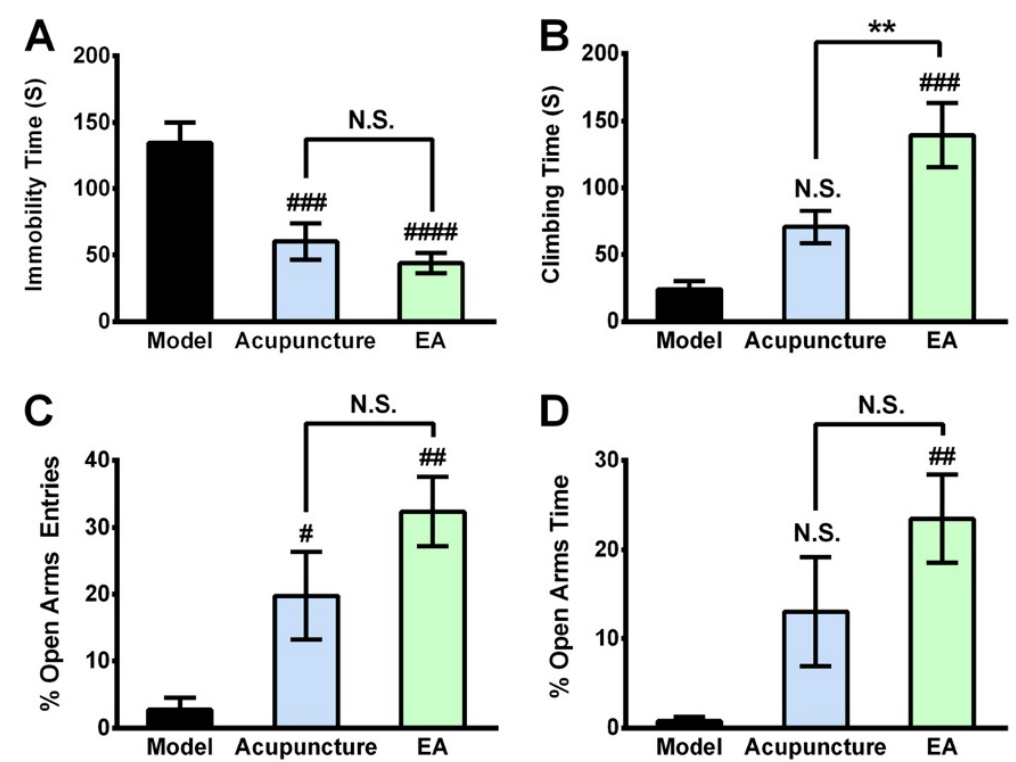

Figure 5 Antidepressant-like effects of EA involved contribution from both acupoint specificity and electrical stimulus. Tests were performed after five treatments. (A) The immobility times in the FST; (B) The climbing time in the FST; (C) \& (D) The percentage of open-arms entries and open-arms time in the EPM. The values are the mean $\pm \mathrm{SE}, \mathrm{n}=8$ or 9 . \# $P<0.05$, \#\# $P<0.01$ (Acupuncture or EA vs. Model). 
highly controversial [21-23], our results are agreement with the relationship between declined NSC proliferation and depression. In addition, there has been broad consensus that the effects of a major antidepressant drugs are closely related to NSC proliferation or neurogenesis $[21,24,25]$. The present study also confirmed EA, as an alternative therapy for depression, may improve the microenvironment, raise the activation of ERK in NSCs, in turn, up-regulate the declined NSC proliferation and play an antidepressant role. Therefore, this paper may provide some new data regarding the neurobiological bases of the etiology, as well as the recovery, of the depression.

The upregulated level of some growth/neuortropic factors in extracellular fluid might account for the antidepressant effects of EA. And a few of studies have indicated that the levels of these growth/neurotrophic factors were raised in response to EA [1,26-28]. In addition, our work and those of others [29-32], show that relief of the depressive disorder by EA coincides with the activation of the ERK signaling pathway. Intracellular pathways elicited by growth/neurotrophic factors typically converge at the MAP kinase cascade level [33]. In addition to neurotrophic factors, several reports have suggested that EA could regulate the expression level of some anti-apoptotic proteins (e.g., Bcl-2), apoptosisassociated proteins (e.g., Bax, TRPM7), some receptors (e.g., glucocorticoid) and/or some neurotransmitters (e.g., 5 -HT) in the hippocampus $[29,34,35]$. These factors also were involved in the activation of ERK signaling pathway in NSCs [36-38]. EA, in all probability, may activate ERK signaling pathways in NSCs through a variety of routes and improve depressive symptoms.

As the in vivo results showed, EA significantly increased the number of BrdU+/Sox $2+$ cells and the p-ERK1/2 level accompanying the amelioration of depression. However, the in-vitro-culture experiments revealed that the extracellular fluid of the hippocampus in EA-treated rats did not up-regulate NSC viability. This result may have been caused by the following reasons. First, the most likely reason is that the CCK- 8 assay was less sensitive for detecting proliferation, compared with the EdU or BrdU method [39]. Also, the in-vivo role of EA may be underlied by intercellular transmission of information, besides the extracellular ingredients [40].

In addition, our results were consistent with some known references which showed that neurosphere formation (or maintenance of NSC properties) and the ERK signaling pathway in NSCs may be correlative [41,42]. This study suggests that EA might confer a neuroprotective hippocampal microenvironment. Future studies focused on the specific neurochemical changes in the microdialysates might also reveal additional molecular mechanisms.
Finally, the mechanisms mediating the antidepressantlike effects could be the extracellular microenvironment in all probability. Antidepressants (including EA) induced hippocampal cells to establish a proliferation-promoting extracellular microenvironment, and roles in this microenvironment contribute to neurogenesis in the NSCs, highly probably via ERK signaling pathways.

\section{Conclusions}

Taken together, we have shown that the proliferative effects of EA treatment on NSCs may be related to the modulation the ERK signaling pathways in the DG. Microdialysates from the hippocampus of EA-treated rats promoted the in vitro growth of neurospheres and induced ERK-signaling-pathway activation. In addition, mechanisms underlying the antidepressant-like effects of EA included both acupoint specificity and electrical stimulus. EA may serve as a potential tool for depression treatment in the future.

\section{Abbreviations}

NSC: Adult neural stem cell; CUS: Chronic unpredictable stress;

EA: Electroacupunture; NGF: Nerve growth factor; BDNF: Brain-derived neurotrophic factor; GDNF: Glial cell line-derived neurotrophic factor; ERK: Extracellular signal-regulated kinase; DG: Dentate gyrus; FST: Forced swimming test; EPM: Elevated plus-maze; PFA: Paraformaldehyde.

\section{Competing interests}

The authors hereby declared that there is no competing interest.

\section{Authors' contributions}

JY and GW guided this study. JY, LY and QL designed the study. LY wrote the protocol, managed the literature searches, undertook the experiment operation and statistical analysis. LY wrote the first draft of the manuscript; $\mathrm{QL}$ and JY helped to revise the manuscript. NY, XZ and QH (these authors contributed equally to this work) contributed to some work of the statistical analysis and have approved the final manuscript.

\section{Acknowledgement}

This project was financially supported by the National Natural Science Foundation of China (No. 30873320, No. 30900426 and No. 81071103) National Basic Research Programme of China (No. 2007CB512303) and the Foundation of Ministry of Education of China (No. 200802460051).

Received: 11 April 2013 Accepted: 23 October 2013

Published: 28 October 2013

\section{References}

1. First M, Gil-Ad I, Taler M, Tarasenko I, Novak N, Weizman A: The effects of fluoxetine treatment in a chronic mild stress Rat model on depressionrelated behavior, brain neurotrophins and ERK expression. J Mol Neurosci 2011, 45(2):246-255.

2. Yun S-J, Park H-J, Yeom M-J, Hahm D-H, Lee H-J, Lee EH: Effect of electroacupuncture on the stress-induced changes in brain-derived neurotrophic factor expression in rat hippocampus. Neurosci Lett 2002, 318:85-88.

3. Lee B, Shim I, Lee HJ, Yang Y, Hahm DH: Effects of acupuncture on chronic corticosterone-induced depression-like behavior and expression of neuropeptide $Y$ in the rats. Neurosci Lett 2009, 453(3):151-156.

4. Liu Q, Yu J, Mi WL, Mao-Ying QL, Yang R, Wang YQ, Wu GC Electroacupuncture attenuates the decrease of hippocampal progenitor cell proliferation in the adult rats exposed to chronic unpredictable stress. Life Sci 2007, 81(21-22):1489-1495.

5. Moret C: Depression: emerging research and treatment approaches. 16-17 January 2003, Paris, France. IDrugs 2003, 6(3):183-186. 
6. Wong ML, Licinio J: Research and treatment approaches to depression. Nat Rev Neurosci 2001, 2(5):343-351.

7. Boscia F, Esposito CL, Crisci AD, Franciscis V, Annunziato L, Cerchia L: GDNF selectively induces microglial activation and neuronal survival in CA1/CA3 Hippocampal regions exposed to NMDA insult through Ret/ERK signalling. PloS one 2009, 4(8):e6486.

8. Sakata H, Niizuma K, Yoshioka H, Kim GS, Jung JE, Katsu M, Narasimhan P, Maier CM, Nishiyama Y, Chan PH: Minocycline-preconditioned neural stem cells enhance neuroprotection after ischemic stroke in rats. J Neurosci 2012, 32(10):3462-3473.

9. Yang J, Pei Y, Pan YL, Jia J, Shi C, Yu Y, Deng JH, Li B, Gong XL, Wang X, et al: Enhanced antidepressant-like effects of electroacupuncture combined with citalopram in a rat model of depression. Evid-Based Complement Alter Med 2013, 2013:107380

10. Hwang IK, Chung JY, Yoo DY, Yi SS, Youn HY, Seong JK, Yoon YS: Effects of electroacupuncture at Zusanli and Baihui on brain-derived neurotrophic factor and cyclic AMP response element-binding protein in the hippocampal dentate gyrus. J Veterin Med Sci 2010, 72(11):1431-1436.

11. Autry AE, Adachi M, Cheng P, Monteggia LM: Gender-specific impact of brain-derived Neurotrophic factor signaling on stress-induced depression-like behavior. Biolog Psych 2009, 66(1):84-90.

12. Hadianfard MJ, Hosseinzadeh Parizi M: A randomized clinical trial of fibromyalgia treatment with acupuncture compared with fluoxetine. Iran Red Cresc Med J 2012, 14(10):631-640.

13. Liu Q: Changes of Adult Hippocampal Neurogenesis during Treatment of Electroacupuncture with Depression Model Rats. Fudan University; Shanghai, China, 2008.

14. Jin Y, Zheng J, Qiong L, Jun W, Yan-qing W, Gen-cheng W: Effect of electroacupuncture on behavioral activities of depression rats. Zhen $\mathrm{Ci}$ Yan Jiu 2006, 1:33-36.

15. Bing L, Qiong L, Liu Y, Na Y, Jin Y, Gencheng W: Changes of behavior during Electro-acupuncture treatment of learned helplessness rats. Sciencepaper Online; 2012.

16. Han C, Li X, Luo H, Li X: Clinical study on electro-acupuncture treatment for 30 cases of mental depression. J Trad Chinese Med 2004, 24(3):172-176.

17. Duan DM, Tu Y, Jiao S, Qin W: The relevance between symptoms and magnetic resonance imaging analysis of the hippocampus of depressed patients given electro-acupuncture combined with Fluoxetine intervention - A randomized, controlled trial. Chin J Integr Med 2011, 17(3):190-199.

18. Yeung AS, Ameral VE, Chuzi SE, Fava M, Mischoulon D: A pilot study of acupuncture augmentation therapy in antidepressant partial and non-responders with major depressive disorder. J Affect Disord 2011, 130(1-2):285-289.

19. Blanco C, Okuda M, Markowitz JC, Liu SM, Grant BF, Hasin DS: The epidemiology of chronic major depressive disorder and dysthymic disorder: results from the National Epidemiologic Survey on Alcohol and Related Conditions. J Clin Psych 2010, 71(12):1645-1656.

20. Kiyohara C, Yoshimasu K: Molecular epidemiology of major depressive disorder. Environ Health Prev Med 2009, 14(2):71-87.

21. Balu DT, Lucki I: Adult hippocampal neurogenesis: regulation, functional implications, and contribution to disease pathology. Neurosci Biobehav Rev 2009, 33(3):232-252.

22. Reif $A$, Fritzen $S$, Finger $M$, Strobel $A$, Lauer $M$, Schmitt $A$, Lesch KP: Neural stem cell proliferation is decreased in schizophrenia, but not in depression. Mol Psych 2006, 11(5):514-522.

23. Vollmayr B, Simonis C, Weber S, Gass P, Henn F: Reduced cell proliferation in the dentate gyrus is not correlated with the development of learned helplessness. Biolog Psych 2003, 54(10):1035-1040.

24. Guo YJ, Zhang ZJ, Wang SH, Sui YX, Sun Y: Notch1 signaling, hippocampal neurogenesis and behavioral responses to chronic unpredicted mild stress in adult ischemic rats. Prog Neuro-Psychopharma Biolog Psych 2009, 33(4):688-694.

25. DeCarolis NA, Eisch AJ: Hippocampal neurogenesis as a target for the treatment of mental illness: a critical evaluation. Neuropharmacol 2010 58(6):884-893.

26. Hisaoka K, Takebayashi M, Tsuchioka M, Maeda N, Nakata Y, Yamawaki S: Antidepressants increase glial cell line-derived Neurotrophic factor production through monoamine-independent activation of protein tyrosine kinase and extracellular signal-regulated kinase in glial cells. J Pharmacol Exper Therapeut 2007, 321(1):148-157.
27. Dong Z-Q, Ma F, Xie H, Wang Y-Q, Wu G-C: Changes of expression of glial cell line-derived neurotrophic factor and its receptor in dorsal root ganglions and spinal dorsal horn during electroacupuncture treatment in neuropathic pain rats. Neurosci Lett 2005, 376(2):143-148.

28. Gureje O, Kola L, Afolabi E: Epidemiology of major depressive disorder in elderly Nigerians in the Ibadan Study of Ageing: a community-based survey. Lancet 2007, 370(9591):957-964.

29. Zhao L, Wang Y, Sun N, Liu X, Li L, Shi J: Electroacupuncture regulates TRPM7 expression through the trkA/PI3K pathway after cerebral ischemia-reperfusion in rats. Life Sci 2007, 81(15):1211-1222.

30. Li J, Li J, Liang F, Hong Y, Wu S, Tang H, Wang H: Electroacupuncture at PC6 (neiguan) improves extracellular signal-regulated kinase signaling pathways through the regulation of neuroendocrine cytokines in myocardial hypertrophic rats. Evid-Based Complement Alter Med 2012, 2012:1-9.

31. Du J, Wang Q, Hu B, Peng Z, Zhao Y, Ma L, Xiong L, Lu Y, Zhu X, Chen S: Involvement of ERK $1 / 2$ activation in electroacupuncture pretreatment via cannabinoid CB1 receptor in rats. Brain Res 2010, 1360:1-7.

32. Yoo S-S, Kerrc CE, Parkc M, Imd D-m, Blinder RA, Park H, Kaptchuk TJ: Neural activities in human somatosensory cortical areas evoked by acupuncture stimulation 败. Complement Therap Med 2007, 15(4):247-254.

33. Gegelashvili G, Dehnes Y, Danbolt NC, Schousboe A: The high-affinity glutamate transporters GLT1, GLAST, and EAAT4 are regulated via different signalling mechanisms. Neurochem Int 2000, 37(2-3):163-170.

34. Liu Z-b, Niu W-m, Yang X-h, X-m N, Wang Y: Effect of "Xiusanzhen"on expression of hippocampal $\mathrm{BCl}-2$ and Bax proteins in Alzheimer disease rats. Zhen Ci Yan Jiu 2011, 36(1):7-11.

35. Zhu Y, Liu Q, Zhuo L-s: Influence of electroacupuncture of "Baihui" (GV 20) and "Sanyinjiao" (SP 6) on hippocampal 5-HT and AChE immuno-activity in chronic depression rats. Zhen Ci Yan Jiu 2009, 34(1):16-20

36. Peng CH, Chiou SH, Chen SJ, Chou YC, Ku HH, Cheng CK, Yen CJ, Tsai TH, Chang YL, Kao CL: Neuroprotection by Imipramine against lipopolysaccharide-induced apoptosis in hippocampus-derived neural stem cells mediated by activation of BDNF and the MAPK pathway. Eur Neuropsychopharma 2008, 18(2):128-140

37. Liu L, Zhao L, She H, Chen S, Wang JM, Wong C, McClure K, Sitruk-Ware R, Brinton RD: Clinically relevant progestins regulate neurogenic and neuroprotective responses in vitro and in vivo. Endocrinol 2010, 151(12):5782-5794.

38. McKelvey L, Gutierrez H, Nocentini G, Crampton SJ, Davies AM, Riccardi CR O'Keeffe GW: The intracellular portion of GITR enhances NGF-promoted neurite growth through an inverse modulation of Erk and NF-kappaB signalling. Biol Open 2012, 1(10):1016-1023.

39. Kang K, Oh SH, Yun JH, Jho EH, Kang JH, Batsuren D, Tunsag J, Park KH, Kim M, Nho CW: A novel topoisomerase inhibitor, daurinol, suppresses growth of HCT116 cells with low hematological toxicity compared to etoposide. Neoplasia 2011, 13(11):1043-1057.

40. Cheng H, Yu J, Jiang Z, Zhang X, Liu C, Peng Y, Chen F, Qu Y, Jia Y, Tian Q, et al: Acupuncture improves cognitive deficits and regulates the brain cell proliferation of SAMP8 mice. Neurosci Lett 2008, 432(2):111-116.

41. Encinas JM, Michurina TV, Peunova N, Park J-H, Tordo J, Peterson DA, Fishell G, Koulakov A, Enikolopov G: Division-coupled astrocytic differentiation and age-related depletion of neural stem cells in the adult hippocampus. Cell Stem Cell 2011, 8(5):566-579.

42. Ma DK, Ponnusamy K, Song MR, Ming GL, Song H: Molecular genetic analysis of FGFR1 signalling reveals distinct roles of MAPK and PLCgamma1 activation for self-renewal of adult neural stem cells. Mol Brain 2009, 2:16.

doi:10.1186/1472-6882-13-288

Cite this article as: Yang et al:: Electroacupuncture upregulates ERK signaling pathways and promotes adult hippocampal neural progenitors proliferation in a rat model of depression. BMC Complementary and Alternative Medicine 2013 13:288. 